\title{
Optimización de las condiciones \\ de crecimiento de la bacteria endófita Klebsiella oxytoca en matraz y en biorreactor
}

Optimization of the growth conditions of the endophytic bacteria Klebsiella oxytoca in flask culture and in a bioreactor

\section{Alexander Schmidt-Durán1, Randall Chacón-Cerdas²}

Fecha de recepción: 17 de setiembre de 2020

Fecha de aprobación: 4 de diciembre de 2020

Schmidt-Durán, A; Chacón-Cerdas, R. Optimización de las condiciones de crecimiento de la bacteria endófita Klebsiella oxytoca en matraz y en biorreactor. Tecnología en Marcha. Vol. 34-4 Octubre-Diciembre 2021. Pág 92-104.

doi) https://doi.org/10.18845/tm.v34i4.5385

1 Instituto Tecnológico de Costa Rica. Costa Rica. Correo electrónico: aschmidt@tec.ac.cr 


\title{
Palabras claves
}

Klebsiella oxytoca, cultivo en matraz, biorreactor, residuos agroindustriales, modelo logístico.

\section{Resumen}

Las bacterias endófitas son aquellas que viven en el interior de las células o los tejidos de plantas superiores sin causar daño. Klebsiella oxytoca es una bacteria endófita promotora del crecimiento vegetal y con efecto biocontrolador, razón por la cual, el objetivo del presente estudio fue determinar las condiciones óptimas de crecimiento de esta bacteria, en matraz y en biorreactor, obtenida de plantas de higo (Ficus carica) en estudios anteriores. Para esto, se evaluó el crecimiento de la biomasa de K. oxytoca en caldo nutritivo bajo diferentes condiciones de agitación y temperatura en matraz, determinando que las condiciones a $200 \mathrm{rpm}$ y $30{ }^{\circ} \mathrm{C}$ fueron las que produjeron una mayor biomasa. Posteriormente, utilizando medio caldo nutritivo en el biorreactor de 3,7 L, se determinó que una agitación de 500 rpm presenta valores de $\mathrm{K}_{\mathrm{L}} \mathrm{a}$ más altos y tiempos de fermentación menores que si se trabaja a 750 rpm. Además, bajo una agitación de 500 rpm, se determinó que medios basados en residuos agroindustriales pueden funcionar como fuentes de crecimiento alternativas para este microorganismo. Por último, el tratamiento de caldo nutritivo a $500 \mathrm{rpm}$ fue modelado mediante el modelo logístico y la ecuación de Pirt, obteniendo, en ambos casos, coeficientes de determinación superiores a 0,95.

\section{Keywords}

Klebsiella oxytoca; flask culture; bioreactor; agro-industrial residues; logistic model.

\begin{abstract}
Endophytic bacteria are characterized for living within plant cells and tissue without causing any harm to the plants. Klebsiella oxytoca is an endophyte that promotes plant growth and also has a bio-controlling effect; therefore, the objective of the current study is to determine the optimal growth conditions, in flask culture as well as in a bioreactor, for this bacteria collected from previous studies performed on fig plants (Ficus carica). To achieve this, biomass growth of $K$. oxytoca flask cultured in nutritive broth was assessed under different agitation and temperature conditions, and it was determined that $200 \mathrm{rpm}$ and $30^{\circ} \mathrm{C}$ produced the highest biomass yield. On the other hand, using a broth culture in a $3.7 \mathrm{~L}$ bioreactor, it was established that $500 \mathrm{rpm}$ presents higher $\mathrm{K}_{\mathrm{L}}$ a values and a shorter fermentation time than when using an agitation speed of $750 \mathrm{rpm}$. Additionally, under an agitation speed of $500 \mathrm{rpm}$, the use of culture media based on agro-industrial residues can provide an alternate source of energy for this microorganism. Finally, the treatment using culture broth at $500 \mathrm{rpm}$ was modeled using a logistic model and Pirt's equation, resulting in both cases, on a coefficient of determination higher than 0.95.
\end{abstract}

\section{Introducción}

Las bacterias pueden crear asociaciones cercanas con las plantas, como por ejemplo patogénicas, epífitas, endófitas, simbióticas, no simbióticas y antagónicas. Estos microorganismos forman grupos diversos filogenéticamente representados por especies pertenecientes en amplia cantidad a diferentes géneros y familias. Cuando se establecen tales vínculos, típicamente se intercambian señales con su hospedero y se desarrollan diversos mecanismos para su adaptación y colonización [1]. 
Los microorganismos endófitos comprenden hongos y bacterias que viven, sin causar daño, en el interior de las células y los tejidos de las plantas superiores, durante una parte de su ciclo de vida bifásico, que alterna entre la planta y el suelo [2] [3]. Principalmente, residen en los espacios intercelulares y muy pocas veces en espacios intracelulares o dentro de los tejidos vasculares [1]. Las bacterias endófitas promueven el crecimiento de las plantas, aumentan la resistencia a enfermedades, contribuyen a la fijación biológica de nitrógeno, interactúan con patógenos y brindan protección contra los mismos [4].

Diversa microbiota promotora del crecimiento vegetal se encuentra tanto en la rizósfera como dentro de las plantas en modo de bacterias endófitas. Entre ellos se encuentran géneros pertenecientes a la familia Enterobacteriaceae como Enterobacter, Klebsiella y Serratia [5] [6] [7]. Klebsiella oxytoca es un microorganismo anaerobio facultativo, Gram negativo que está presente en una variedad de fuentes ambientales como el suelo, la vegetación y el agua [8]. Se caracteriza por poseer una prominente cápsula de polisacáridos y la presencia de adhesinas [9] [10].

Klebsiella oxytoca ha sido utilizada con fines biotecnológicos en diversas áreas, presenta potencial biorremediador, pues degrada hidrocarburos [11], compuestos orgánicos hidrofóbicos [12], sustancias tóxicas como el cianuro [13], así como insecticidas organoclorados [14], nitrilos [15], ácidos benzoicos [16], colorantes azoicos [17] y el fármaco diclofenaco [18]. Además, se ha reportado la producción de bioelectricidad en conjunto con la degradación de ácidos grasos [19], ha sido capaz de llevar a cabo la desulfuración termofílica de dibenzotiofenos [20], entre otras muchas aplicaciones [21] [22] [23].

Como bacteria endófita, esta especie ha promovido el crecimiento vegetal debido a su capacidad de fijar nitrógeno y de solubilizar el fósforo [24] [25] [26], produce auxinas [27] [26] y, además, exhibe antagonismo contra Fusarium oxysporum [28], Pythium aphanidermatum [29] y Gaeumannomyces graminis [30]. También ha presentado potencial como inoculante microbiano para biofortificación con selenio [30].

Por esta razón, el objetivo de esta investigación fue determinar las mejores condiciones de cultivo en matraz y en biorreactor de 3,7 L para el crecimiento de la bacteria endófita $K$. oxytoca, aislada de plantas de higo (Ficus carica) como potencial microorganismo de múltiples aplicaciones biotecnológicas.

\section{Metodología}

\section{Obtención de la bacteria endófita Klebsiella oxytoca}

La bacteria Klebsiella oxytoca se obtuvo de crioviales conteniendo $20 \%$ de glicerol y $80 \%$ de medio caldo nutritivo mantenidos a $-80{ }^{\circ} \mathrm{C}$, la cual fue previamente aislada de hojas de higo Ficus carica L. de la variedad "Brown Turkey" [31] e identificada molecularmente por Alvarado-Marchena y colaboradores [32]. Una asada del criovial, previamente descongelado a temperatura ambiente por 15 min, se inóculo en una placa Petri con medio agar nutritivo. Después de $24 \mathrm{~h}$ de crecimiento, se tomó una colonia y fue rayada en otra placa del mismo medio de cultivo.

\section{Cultivo en matraz}

Posteriormente, se realizó un inóculo de la bacteria. En matraces de $250 \mathrm{ml}$, conteniendo $50 \mathrm{ml}$ de medio caldo nutritivo, se colocó una colonia de la bacteria. Se dejó creciendo el microorganismo a 100 rpm durante $18 \mathrm{~h}$. Al cultivo bacteriano en matraz se le realizó un conteo celular mediante cámara de Neubauer y se agregó el volumen necesario para realizar el ensayo de optimización de condiciones de crecimiento en matraz, empezando con una concentración 
inicial de 1,0 × $10^{6} \mathrm{cél} / \mathrm{ml}$. El ensayo se realizó con dos factores diferentes, agitación a 100, 150, 200 y 250 rpm y temperatura a 30 y $37^{\circ} \mathrm{C}$, determinando el conteo celular mediante cámara de Neubauer a las $24 \mathrm{~h}$, tomando una muestra de $1 \mathrm{ml}$ y realizando las diluciones requeridas de acuerdo a la concentración celular. Para cada tratamiento se realizaron tres repeticiones. A los resultados obtenidos se les realizó un análisis de varianza y una prueba de Tukey usando el programa estadístico Minitab® [33].

\section{Crecimiento en biorreactor bajo diferentes agitaciones}

Se realizó fermentaciones en un biorreactor R'ALFs de 3,7 I (Bioengineering $®$ ). El equipo se utilizó a $30{ }^{\circ} \mathrm{C}$, con caldo nutritivo adicionando $5 \mathrm{~g} / \mathrm{l}$ de glucosa, un $\mathrm{pH}$ de 7.0 , un impulsor tipo propela marina y se probaron dos agitaciones diferentes: 500 y $750 \mathrm{rpm}$. Para cada ensayo se determinó la $\mathrm{K}_{\mathrm{L}}$ a por medio del método dinámico.

El pre-inóculo se realizó bajo las condiciones optimizadas con anterioridad, en el cual se inoculó una concentración requerida para iniciar la fermentación con 1,0 × 106 cél/ml de la bacteria. Se tomaron muestras cada 1 o 2 h y se analizó la biomasa mediante cámara de Neubauer y densidad óptica a una absorbancia de $600 \mathrm{~nm}$ y el consumo de glucosa mediante el método de Trinder [34].

\section{Prueba con residuos agroindustriales}

Bajo las mismas condiciones de inóculo descritas anteriormente y la agitación determinada en el ensayo anterior, se procedió a realizar las fermentaciones mediante dos medios de cultivo basados en residuos agroindustriales y un tratamiento control, correspondiente al medio caldo nutritivo suplementado con $5 \mathrm{~g} / \mathrm{l}$ de glucosa. Los medios basados en residuos agroindustriales fueron procesados de cáscaras de piña y cáscaras de papaya. Para la elaboración de los medios de cultivo, los residuos se colocaron en una proporción 1:1 con agua destilada y se licuaron durante 10 min hasta su homogenización. Posteriormente, se elaboró el medio disolviendo los residuos homogenizados en una proporción 1:10 v/v con agua destilada, el caldo obtenido se calentó hasta ebullición durante 10 min y luego se filtró para eliminar los residuos sólidos. El filtrado fue el medio de cultivo utilizado en el biorreactor y se le determino la $\mathrm{K}_{\mathrm{L}}$ a mediante el método dinámico. El biorreactor se inoculó con 1,0 × $10^{6} \mathrm{cél} / \mathrm{ml}$ y cada hora, durante 13 horas, se tomaba una muestra en donde se le determinó la biomasa mediante cámara de Neubauer.

\section{Modelaje de la cinética de crecimiento}

A las mejores condiciones de crecimiento se les realizó un modelado primario del crecimiento de la biomasa mediante el modelo logístico [35], dado por la ecuación matemática:

$$
\frac{d X}{d t}=\mu_{\max } X\left(1-\frac{X}{X_{\max }}\right)
$$

Donde $\mathrm{X}_{\max }$ es la biomasa máxima, $\mathrm{X}$ es la biomasa en el tiempo y $\mu_{\max }$ es la velocidad máxima de crecimiento del microorganismo. La integración de la ecuación 1 usando $X=X_{0}(t=0)$ da como resultado la ecuación

$$
X(t)=\frac{X_{0} e^{\mu_{\max } t}}{1-\frac{X_{0}}{X_{\max }}\left(1-e^{\mu_{\max } t}\right)}
$$


Además, al consumo de sustrato se le realizó un modelado a través de la ecuación de Pirt [36] [37], dado por la ecuación matemática:

$$
\frac{1}{Y x / s}=\frac{1}{Y x / s \max }+\frac{m_{s}}{\mu}
$$

Donde $Y_{x / s}$ es el rendimiento de la biomasa sobre el sustrato, $Y_{x / s}$ max es el rendimiento de la biomasa sobre el sustrato máximo y $\mathrm{m}_{\mathrm{s}}$ es el coeficiente de mantenimiento. Si se sustituye la ecuación 2 en la 3, se obtiene la siguiente ecuación del modelo:

$$
S(t)=S_{0}-\frac{1}{Y x / s}\left(\frac{X_{0} e^{\mu_{\max } t}}{1-\frac{X_{o}}{X_{\max }}\left(1-e^{\mu_{\max } t}\right)}-X_{0}\right)-\frac{m_{S} X_{\max }}{\mu_{\max }} \ln \left(1-\frac{X_{0}}{X_{\max }}\left(1-e^{\mu_{\max } t}\right)\right)
$$

\section{Resultados y discusión}

\section{Cultivo en matraz}

En el cuadro 1 se observa la clasificación según la prueba de Tukey aplicada a los diferentes tratamientos de optimización de las condiciones de crecimiento de K. oxytoca en matraz. Los datos presentaron homocedasticidad ( $p>0,050)$, aleatoriedad y normalidad $(p>0,050)$. El análisis de varianza determinó que existen diferencias significativas en la temperatura $(p=0,000)$ y la agitación $(p=0,000)$, además, las variables presentaron interacción entre ellas $(p=0,000)$.

Cuadro 1. Prueba de rangos de Tukey de los diferentes tratamientos realizados en matraz.

\begin{tabular}{|c|c|}
\hline Tratamiento & Promedio Biomasa $(\mathrm{cél} / \mathrm{ml})^{\star}$ \\
\hline $100 \mathrm{rpm}$ a $30^{\circ} \mathrm{C}$ & $1,2 \times 10^{\circ} \mathrm{bc}$ \\
\hline $150 \mathrm{rpm}$ a $30^{\circ} \mathrm{C}$ & $1,8 \times 10^{9} \mathrm{ab}$ \\
\hline $200 \mathrm{rpm}$ a $30^{\circ} \mathrm{C}$ & $2,4 \times 10^{9} \mathrm{a}$ \\
\hline $250 \mathrm{rpm}$ a $30^{\circ} \mathrm{C}$ & $7,1 \times 10^{8} \mathrm{~d}$ \\
\hline $100 \mathrm{rpm}$ a $37^{\circ} \mathrm{C}$ & $6,9 \times 10^{8} \mathrm{~d}$ \\
\hline $150 \mathrm{rpm}$ a $37^{\circ} \mathrm{C}$ & $1,6 \times 10^{9} \mathrm{ab}$ \\
\hline $200 \mathrm{rpm}$ a $37^{\circ} \mathrm{C}$ & $8,0 \times 10^{8} \mathrm{~d}$ \\
\hline $250 \mathrm{rpm} \mathrm{a} 37^{\circ} \mathrm{C}$ & $8,9 \times 10^{8} \mathrm{~cd}$ \\
\hline
\end{tabular}

*Letras diferentes significa que presentan diferencias estadísticas significativas.

La temperatura afecta la estabilidad y la actividad biológica de diferentes moléculas de los microorganismos, por lo que cada microorganismo tiene un rango de temperatura de crecimiento y una temperatura en donde alcanza su velocidad máxima de crecimiento [38]. Así, diferentes temperaturas permiten la obtención de diferentes rendimientos de biomasa y de productos. Klebsiella oxytoca presenta un amplio rango de temperaturas de crecimiento junto a otros microorganismos de su misma familia, que pueden ir desde los $25^{\circ} \mathrm{C}$ hasta los $37^{\circ} \mathrm{C}$ de manera óptima [39]. Bajo esta premisa, se evaluó el efecto de la temperatura en el crecimiento de $\mathrm{K}$. oxytoca, obteniendo que una temperatura de $30^{\circ} \mathrm{C}$ generó una mayor biomasa de manera significativa que la obtenida a $37^{\circ} \mathrm{C}$. 
Este fenómeno ya se ha reportado en otras cepas de este mismo microorganismo, en donde una cepa aislada de suelos costeros, presentó un crecimiento que fue casi el doble en términos de absorbancia, a temperaturas de $30^{\circ} \mathrm{C}$ con respecto a la misma cepa en una temperatura de $37^{\circ} \mathrm{C}$, no obstante, la producción de los compuestos de interés en esta investigación (2,3 butanediol y acetoína), fueron mayores a $37^{\circ} \mathrm{C}$ [40] sin embargo, también hay reportes de crecimiento a $37^{\circ} \mathrm{C}$ de este microorganismo, para la producción de los mismos compuestos mencionados [41]. Por otro lado, se reporta la degradación de carbendazim de una cepa silvestre de K. oxytoca a temperatura ambiente, alcanzando concentraciones de 3,5 × 107 cél/ $\mathrm{ml}$, utilizando a este compuesto como fuente de carbono [42], mientras que en otro estudio de biorremediación, se ha utilizado este microorganismo a $30{ }^{\circ} \mathrm{C}$ para la degradación de cianuro [43].

En relación con la agitación, esta permite la homogenización de la biomasa, la dispersión de las burbujas de aire, del medio de cultivo, $\mathrm{pH}$ y del calor en medios líquidos [38]. Diferentes autores han reportado el crecimiento de K. oxytoca en condiciones en matraz bajo diferentes revoluciones por minuto, las cuales fueron $80 \mathrm{rpm}$ [42], $150 \mathrm{rpm}$ [40] [41] y $200 \mathrm{rpm}$ [44] [45], lo que provocó que se evaluaran cuatro agitaciones diferentes para la producción de biomasa. Bajo las variables analizadas, se pudo determinar que existe una interacción entre las variables temperatura y agitación, determinando que, a $200 \mathrm{rpm}$ con una temperatura de $30{ }^{\circ} \mathrm{C}$, es la condición óptima de los tratamientos evaluados, para el crecimiento de K. oxytoca.

\section{Prueba en biorreactor con diferentes agitaciones}

En la figura 1 se observa la cinética de crecimiento por conteo celular (1a) y densidad óptica (1b) de K. oxytoca bajo dos condiciones diferentes de agitación. En ambos casos se observa que las cinéticas a 500 rpm llegan a fase exponencial y fase estacionaria en un menor tiempo. Sin embargo, a las 13 horas, ambas cinéticas alcanzan concentraciones de 3,0 × 109 cél/ml. Además, se denota el consumo de glucosa por parte del microorganismo (1c), en la que el tratamiento a 500 rpm agota el sustrato a las 11 h, mientras que el tratamiento a 750 rpm requirió $12 \mathrm{~h}$.

Se realizó un ensayo con diferentes agitaciones de 100, 200, 300 y 400 rpm y una estrategia de dos agitaciones (300 rpm durante 15 h y 200 rpm las restantes 15 h) en la fermentación de $K$. oxytoca para la producción de 2,3 butanediol [44]. Los autores obtuvieron una mayor cantidad de biomasa al aumentar la agitación, pasando de 4,3 g/l de peso seco a $100 \mathrm{rpm}$, hasta una concentración de 5,2 g/l de peso seco a los 400 rpm. Sin embargo, la concentración máxima de butanediol se dio a los 200 rpm. La estrategia de dos agitaciones arrojó datos de 5,0 g/l de peso seco y la máxima concentración de butanediol de todos los tratamientos realizados. Por otro lado, en una fermentación con una cepa ingenierizada de K.oxytoca a $400 \mathrm{rpm}$ se obtuvó concentraciones cercanas a los $9 \mathrm{~g} / \mathrm{l}$, utilizando glicerol puro y mayores a $13 \mathrm{~g} / \mathrm{l}$ en fermentaciones con glicerol crudo [45].

De acuerdo con los resultados anteriores, se denota que existe una relación entre la biomasa y la velocidad de agitación, cuando estas son menores a los 400 rpm, sin embargo, es posible que exista una velocidad de agitación máxima, en la cual, ya no exista un mejoramiento en la biomasa obtenida. En este caso particular, la biomasa obtenida en la fermentación en el biorreactor a 500 rpm es mayor que las obtenidas en los tratamientos en matraz, y en un menor tiempo de fermentación, sin embargo, al aumentar la velocidad de agitación, esta no provocó un aumento de la biomasa, más bien, se da un aumento en el tiempo de fermentación, específicamente en la fase lag. Esta fase se caracteriza por ser una fase de adaptación del microorganismo al medio de cultivo y a las condiciones de crecimiento y su duración es variable dependiendo de la fisiología de las células y su grado de adaptación a las condiciones del 
cultivo [38], pudiendo significar que las células bacterianas del inóculo que se encontraban en condiciones de crecimiento de 200 rpm, al adaptarse a las nuevas condiciones de agitación de 750 rpm, provocaran un mayor tiempo de adaptación, alargando el tiempo de la fermentación.
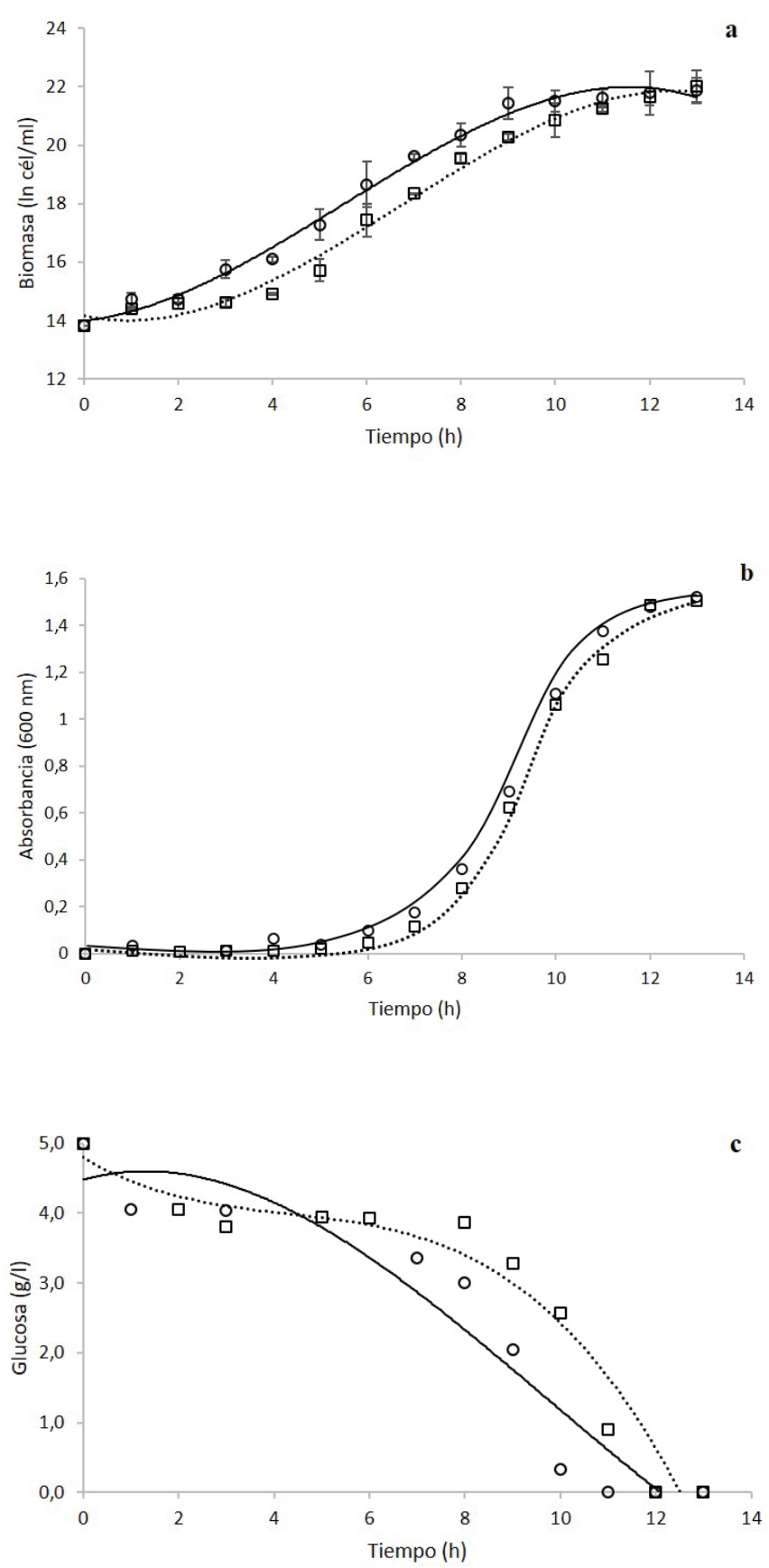

Figura 1. Cinéticas de crecimiento de Klebsiella oxytoca en biorreactor a 500 rpm (círculo con línea continua) y a 750rpm (cuadrados con línea discontinua de puntos) por conteo celular en cámara de Neubauer (a), densidad óptica (b) y consumo de glucosa (c).

Con respecto al coeficiente volumétrico de transferencia de masa, la $\mathrm{K}_{\mathrm{L}}$ a para el tratamiento a 500 rpm fue de 46,352 h-1, mientras que el tratamiento a 750 rpm presentó un valor de 25,808 $\mathrm{h}^{-1}$. Es importante señalar que la transferencia de oxígeno constituye un factor muy importante 
en el crecimiento de los microorganismos y el escalado en fermentaciones aerobias, ya que el oxígeno tiende a ser muchas veces un compuesto limitante, debido a su baja solubilidad en soluciones acuosas, por lo que frecuentemente se emplean alternativas en las condiciones de operación como cambios en la velocidad de agitación, el flujo de aireación o el sistema de distribución de aire [46]. En este caso particular, se obtuvo que a mayor coeficiente de transferencia de oxígeno hay un mayor consumo de este por parte de Klebsiella oxytoca (datos no mostrados), pero en ninguno de los dos tratamientos el oxígeno fue un factor limitante, probablemente por ser un microorganismo anaerobio facultativo y tener una tasa de respiración baja. Además, se denota que una mayor velocidad de agitación, provocó un menor valor de $\mathrm{K}_{\mathrm{L}} \mathrm{a}$. Esto concuerda con los resultados obtenidos por [47] en donde encontraron que, en un biorreactor de tres litros, el valor de la $\mathrm{K}_{\llcorner}$a aumentaba hasta llegar a los $618 \mathrm{rpm}$, valor en el cual, el análisis de superficie generaba la meseta y los valores de $\mathrm{K}_{\llcorner}$a tendían a disminuir. Esto puede ser provocado porque la velocidad de agitación ya no provoca una mejor distribución ni un menor tamaño de las burbujas adentro del biorreactor, sino más bien, provoca coalescencias de la misma, disminuyendo los valores de $\mathrm{K}_{\mathrm{L}} \mathrm{a}$.

Además, de acuerdo con los parámetros de la fermentación obtenidos, los valores de velocidad de crecimiento y el tiempo de duplicación en ambas agitaciones son muy parecidos, sin embargo, a 500 rpm se obtiene un rendimiento mayor, logrando formar una generación más de células en una hora menos de fermentación, por lo que se selecciona esa agitación como la óptima para el crecimiento de K. oxytoca (cuadro 2).

Prueba con residuos agroindustriales

Los valores del coeficiente volumétrico de transferencia de masa fueron altos para el medio caldo nutritivo $\left(46,352 \mathrm{~h}^{-1}\right)$ y para el medio caldo piña $\left(37,023 \mathrm{~h}^{-1}\right)$, no así para el medio de caldo papaya cuyo valor es bastante menor en comparación a los otros tratamientos (14,579 $\mathrm{h}^{-1}$ ). La $\mathrm{K}_{\mathrm{L}}$ a en el biorreactor, es afectada por factores como las propiedades del medio de cultivo y del gas a utilizar, la geometría del biorreactor, las condiciones de operación y el tipo de microorganismo [46], por lo que, en este caso, las diferencias en los valores obtenidos se relacionan con la densidad, viscosidad y otras propiedades propias de los medios de cultivo, que limitaron la transferencia de oxígeno, con respecto al caldo nutritivo. Además, del mismo modo que en el ensayo de agitación, se observó que al tener un mayor valor de $\mathrm{K}_{\mathrm{L}} \mathrm{a}$, la tasa de respiración del microorganismo fue mayor (datos no mostrados), seguramente debido a que el microorganismo es anaerobio facultativo [8].

Además, en la figura 2 se observa las cinéticas de crecimiento por conteo celular. Se evidencia que K. oxytoca logró alcanzar concentraciones de $3,3 \times 10^{9}$ cél/ml, mientras que el medio caldo piña llegó una concentración máxima de $2,3 \times 10^{8}$ cél/ml y el medio caldo papaya a una concentración de 5,4 × $10^{8} \mathrm{cél} / \mathrm{ml}$. Se ha demostrado que este microorganismo es capaz de crecer de manera óptima bajo diferentes fuentes de carbono como lo son la glucosa, galactosa, fructosa, xilosa y sucrosa, con rendimientos cercanos entre sí [40] es decir, puede utilizar tanto azúcares reductores como no reductores.

El medio caldo piña y caldo papaya se elaboraron a partir de residuos agroindustriales de cáscaras con restos de la fruta, el análisis mediante el método de Trinder determinó que el caldo piña contenía 1,67 g/l de glucosa, el medio papaya contenía 1,57 g/l, mientras que al caldo nutritivo contenía $5 \mathrm{~g} / \mathrm{l}$ de glucosa. Sin embargo, se reporta que, el corazón de la piña contiene 0,113 g de azúcares reductores por cada gramo de fruto, de los cuales 0,0114 g de éstos corresponden a glucosa [48]. Mientras que para la papaya se reportan 0,0178 gramos de azúcares reductores por cada gramo de la fruta [49]. 


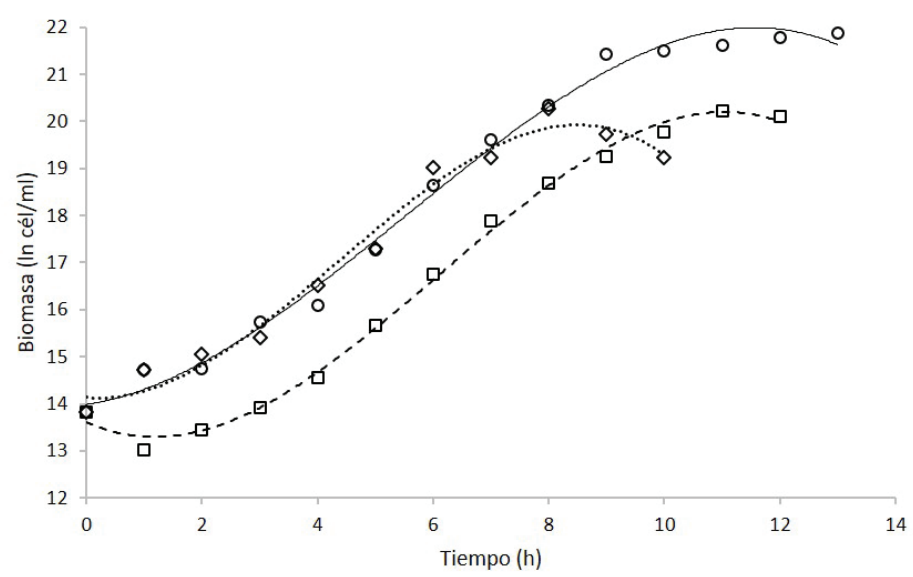

Figura 2. Cinéticas de crecimiento de Klebsiella oxytoca en biorreactor en medio de cultivo caldo nutritivo (círculos con línea continua), caldo piña (cuadrados con línea discontinua de puntos) y caldo papaya (rombos con línea discontinua de rayas) mediante el conteo celular en cámara de Neubauer.

A pesar de que se hace necesario realizar una cuantificación de los azúcares totales y azúcares reductores de ambos residuos agroindustriales, o en su defecto de los medios elaborados, con el fin de comprender a plenitud las diversas fuentes de carbono presentes en el medio de cultivo, así como su concentración, se evidencia que los residuos agroindustriales utilizados presentan una combinación de azúcares, algunos de los cuales son reductores, que pueden ser utilizados como fuente de carbono en fermentaciones de Klebsiella oxytoca, sin embargo, en este caso en particular, los medios basados en residuos agroindustriales presentaron crecimientos menores. La optimización de estos medios, podría permitir aumentar la biomasa de K. oxytoca y, por ende, ser utilizados como alternativa a los medios sintéticos.

Los parámetros obtenidos en las fermentaciones permiten evidenciar que el caldo nutritivo tuvo valores de crecimiento más altos, además que se obtuvieron una menor cantidad de generaciones con tiempos de duplicación más altos (cuadro 2) en comparación con los valores obtenidos en los medios de cultivo basados en residuos agroindustriales.

Cuadro 2. Comparación de parámetros en el biorreactor de 3,7 I bajo diferentes velocidades de agitación y medios de cultivo.

\begin{tabular}{|c|c|c|c|c|c|}
\hline \multirow[t]{2}{*}{ Fermentación } & \multicolumn{5}{|c|}{ Parámetros } \\
\hline & $\mu\left(h^{-1}\right)$ & $T_{d}(h)$ & Generaciones & $\begin{array}{c}\mathrm{Yx} / \mathrm{s} \\
\left(\text { cél } \mathrm{g} \mathrm{g}^{-1} \mathrm{ml}^{-1}\right)^{1}\end{array}$ & $\begin{array}{l}\text { Tiempo de fermentación } \\
\text { (h) }{ }^{2}\end{array}$ \\
\hline $\begin{array}{c}\text { Caldo nutritivo a } 750 \\
\text { rpm }\end{array}$ & 0,99 & 0,72 & $8,5 \approx 9$ & $6,3 \times 10^{8}$ & 10 \\
\hline $\begin{array}{c}\text { Caldo nutritivo a } 500 \\
\text { rpm }\end{array}$ & 0,96 & 0,70 & $9,7 \approx 10$ & $8,1 \times 10^{8}$ & 9 \\
\hline $\begin{array}{l}\text { Caldo piña a } 500 \\
\text { rpm }\end{array}$ & 0,87 & 0,80 & $7,5 \approx 8$ & - & 8 \\
\hline $\begin{array}{c}\text { Caldo papaya a } 500 \\
\text { rpm }\end{array}$ & 0,89 & 0,78 & $7,7 \approx 8$ & - & 9 \\
\hline Modelo ${ }^{3}$ & 1,16 & 0,60 & $10,1 \approx 10$ & $1,3 \times 10^{9}$ & - \\
\hline
\end{tabular}

${ }^{1}$ Rendimiento fue expresado en cél/ml de biomasa / g/l de glucosa

${ }^{2}$ Tiempo de fermentación para llegar a fase estacionaria 3Parámetros obtenidos se determinaron mediante los datos obtenidos del modelo logístico y la ecuación de Pirt aplicados al tratamiento de caldo nutritivo a 500 rpm. 


\section{Modelaje de la cinética de crecimiento}

Con los datos obtenidos, se seleccionó el tratamiento de caldo nutritivo a 500 rpm para realizar el modelaje cinético. De acuerdo con los datos del modelo logístico (figura 3a) (ecuación 2), el coeficiente de determinación del modelo con respecto a los datos experimentales fue de un 97,99\%, dado por la regresión lineal:

Modelo logístico $($ In cél/ml) = 0,9236 *(Biomasa experimental In cél/ml) + 1,7626.

Mientras que, para el consumo de glucosa, la ecuación de Pirt (ecuación 4) respecto a los datos experimentales obtuvo un coeficiente de determinación de 95,98\% (figura 3b), expresado mediante la regresión lineal:

Ecuación de Pirt $(g / l)=0,9992 *(G l u c o s a$ experimental $g / l)$ - 0,1501.

Además, de acuerdo a los datos del modelo, se obtuvieron los parámetros más alto de la velocidad de crecimiento y rendimiento de la biomasa sobre el sustrato, con el menor tiempo de duplicación. Sin embargo, al final llegan a una cantidad similar de generaciones en la fermentación (cuadro 2). Se logró determinar que, en este caso, ambos modelos logran predecir el comportamiento de los datos experimentales.
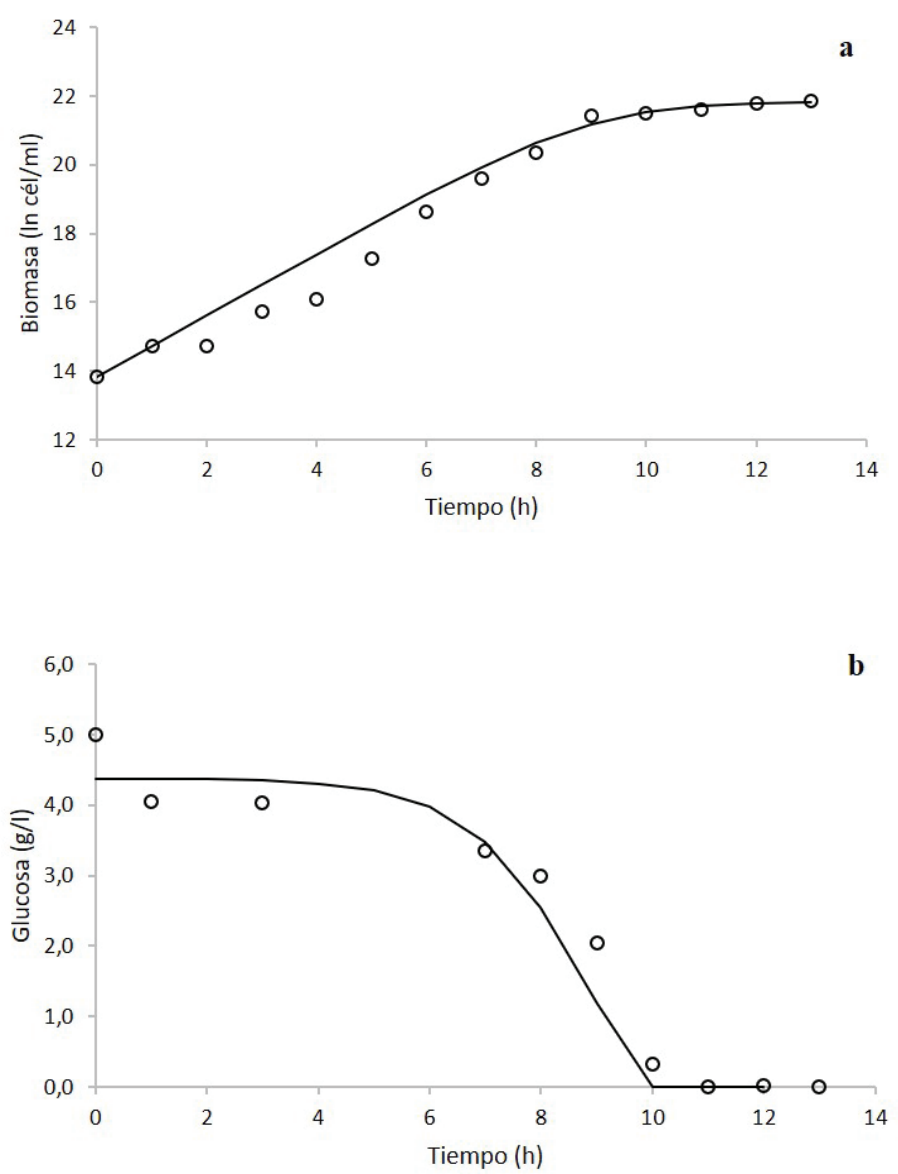

Figura 3. Modelaje de la cinética de crecimiento (a) y consumo de glucosa (b) de Klebsiella oxytoca en biorreactor en medio de cultivo caldo nutritivo a 500 rpm mediante el modelo logístico y la ecuación de Pirt, respectivamente. Los círculos son los datos experimentales y la línea continua son los datos del modelo. 


\section{Conclusiones}

Los cambios en las condiciones de crecimiento de temperatura y agitación para Klebsiella oxytoca en matraz provocaron diferencias significativas en la biomasa final obtenida, evidenciando que estos factores son importantes de optimizar para la producción de biomasa de este microorganismo. Sin embargo, al evaluar velocidades de agitación en el biorreactor, se denotó que este parámetro en altas velocidades (superiores a 500 rpm) no mejora el coeficiente volumétrico de transferencia de masa ni las concentraciones finales de biomasa, más bien, produce un aumento en el tiempo de fermentación del microorganismo. Por otro lado, los medios de cultivo basados en residuos agroindustriales pueden ser utilizados para el crecimiento de Klebsiella oxytoca debido a su capacidad de utilizar azúcares reductores y no reductores como fuentes de carbono. A pesar de que los valores de biomasa son inferiores al medio definido, se recomienda hacer estudios de la composición química de los residuos agroindustriales que permitan optimizar el medio, al suplementarse con otros componentes, lo que podría aumentar los rendimientos y las biomasas obtenidas. Por último, se logró evidenciar que el modelo logístico y la ecuación de Pirt pueden predecir el comportamiento de las cinéticas de crecimiento y consumo de glucosa de Klebsiella oxytoca en biorreactor.

\section{Referencias}

[1] A. Perez, J. Rojas, y J. Fuentes, "Diversidad de bacterias endófitas asociadas a raíces del pasto colosuana (Bothriochloa pertusa) en tres localidades del departamento de sucre, Colombia," Acta Biológica Colombiana, vol. 15, no. 2, pp. 219-228, 2010.

[2] I. Afzal, Z. K. Shinwari, S. Sikandar, y S. Shahzad, "Plant beneficial endophytic bacteria: Mechanisms, diversity, host range and genetic determinants," Microbiological Research, vol. 221, pp. 36-49, 2019.

[3] G. Santoyo, G. Moreno-Hagelsieb, M. D. C. Orozco-Mosqueda, y B. R. Glick, "Plant growth-promoting bacterial endophytes," Microbiological Research, vol. 183, pp. 92-99, 2016.

[4] A. Perez, y L. Chamorro, "Bacterias endófitas: una alternativa biológica para el control de Burkholderia glumae en el cultivo del arroz en Colombia," Revista Colombiana de Ciencia Animal, vol. 4, no. 1, pp. 172-184, 2012.

[5] H. Zhang, Q. Zhang, S. Chen, Z. Zhang, J. Song, Z. Long, Y. Yu, y H. Fang, "Enterobacteriaceae predominate in the endophytic microbiome and contribute to the resistome of strawberry," Science of The Total Environment, vol. 727, pp. 1-9, 2020.

[6] M. Bind, y S. Nema, "Isolation and Molecular Characterization of Endophytic Bacteria from Pigeon Pea Along with Antimicrobial Evaluation against Fusarium udum," Appl Microbiol Open Access, vol. 5, no. 163, pp. 1-12, 2019.

[7] A. Sánchez-Bautista, C. de León-García, S. Aranda-Ocampo, E. Zavaleta-Mejía, y C. Nava-Díaz, "Bacterias endófitas de la raíz en líneas de maíces tolerantes y susceptibles a sequía," Revista Mexicana de Fitopatología, pp. 35-55, 2017.

[8] L. Singh, M. Cariappa, y M. Kaur, "Klebsiella oxytoca: An emerging pathogen?," Medical Journal Armed Forces India, vol. 72, pp. 1-3, 2016.

[9] M. D. Alcántar-Curiel, D. Blackburn, Z. Saldaña, C. Gayosso-Vázquez, N. lovine, M. A. de la Cruz, y J. A. Girón, "Multi-functional analysis of Klebsiella pneumoniae fimbriae types in adherence and biofilm formation," Virulence, vol. 4, no. 2, pp. 129-138, 2013.

[10] A. Ghasemian, A. Mobarez, S. Peerayeh, y A. B. Abadi, "The association of surface adhesin genes and the biofilm formation among Klebsiella oxytoca clinical isolates," New Microbes and New Infections, vol. 27, pp. 36-39, 2019.

[11] A. Nava, A. Ronquillo, A. Cabrera, D. Silva, K. Pérez, y C. García, "Klebsiella oxytoca: El futuro de la biorremediación," Revista Materia, Ciencia y Nanociencia, vol. 2, no. 1, pp. 9-14, 2019.

[12] D. Zhang, L. Lu, H. Zhao, M. Jin, T. Lü, y J. Lin, "Application of Klebsiella oxytoca Biomass in the Biosorptive Treatment of PAH-Bearing Wastewater: Effect of PAH Hydrophobicity and Implications for Prediction," Water, vol. 10, no. 6, pp. 1-14, 2018.

[13] N. H. Avcıoğlu, y I. S. Bilkay, "Cyanide Removal in Electroplating, Metal Plating and Gold Mining Industries' Wastewaters by Using Klebsiella pneumoniae and Klebsiella oxytoca Species” vol. 78, no. 1, pp. 5-10, 2019. 
[14] M. C. Ifediegwu, K. C. Agu, N.S. Awah, A. E. Mbachu, C. B. Okeke, C. G. Anaukwu, P. O. Uba, U.C. Ngenegbo, y C. M. Nwankwo, "Isolation, Growth and Identification of Chlorpyrifos Degrading Bacteria from Agricultural Soil in Anambra State, Nigeria," Universal Journal of Microbiology Research, vol. 3, no. 4, pp. 46-52, 2015.

[15] C. Liu, K. Yuan, R. P. Chen, M. J. Chen, y L. Yu, "Biodegradation kinetics of nitriles with easily degradable substrate by Klebsiella oxytoca GS-4-08," International Biodeterioration \& Biodegradation, vol. 118, pp. 95-101, 2017.

[16] I. H. Aljundi, K. M. Khleifat, A. M. Khlaifat, A. M. Ibrahim, K. A. Tarawneh, y S. A. Tarawneh, "Biodegradation of 2-Chlorobenzoic Acid by Klebsiella oxytoca: Mathematical Modeling and Effect of Some Growth Conditions," Industrial \& Engineering Chemistry Research, vol. 49, no. 16, pp. 7159-7167, 2010.

[17] A. Khalid, J. Arshad, S. Mahmood, I. Aziz, y M. Arshad, "Effect of Chromium Forms on the Biodegradation of Reactive Black-5 Azo Dye by Psychrobacter and Klebsiella species," International Journal of Agriculture and Biology, vol. 17, no. 6, pp. 1260-1264, 2015.

[18] K. Stylianou, E. Hapeshi, M. I. Vasquez, D. Fatta-Kassinos, y I. Vyrides, "Diclofenac biodegradation by newly isolated Klebsiella sp. KSC: Microbial intermediates and ecotoxicological assessment," Journal of Environmental Chemical Engineering, vol. 6, no. 2, pp. 3242-3248, 2018.

[19] A. Kingsly, D. Jothinathan, y W. R. Thilagaraj, "Degradation of oleic acid and simultaneous bioelectricity production by Klebsiella oxytoca ADR 13," Energy Sources, Part A: Recovery, Utilization, and Environmental Effects, vol. 39, no. 9, pp. 874-882, 2017.

[20] S. Bhatia, y K. Sharma, "Thermophilic desulfurization of dibenzothiophene and different petroleum oils by Klebsiella sp. 13T," Environ Sci Pollut Res, vol. 19, pp. 3491-3497, 2012.

[21] W. Meng, Y. Zhang, M. Cao, W. Zhang, C. Lü, C. Yang, C. Gao, P. Xu, y C. Ma, "Efficient 2,3-butanediol production from whey powder using metabolically engineered Klebsiella oxytoca," Microbial Cell Factories, vol. 19, no. 162, 2020.

[22] J. Huang, S. Zhu, L. Zhao, L. Chen, M. Du, C. Zhang, y S. T. Yang, "A novel ß-galactosidase from Klebsiella oxytoca ZJUH1705 for efficient production of galacto-oligosaccharides from lactose," Applied Microbiology and Biotechnology, vol. 104, no. 14, pp. 6161-6172, 2020.

[23] N. Li, H. Chou, L. Yu, y Y. Xu, "Cadaverine production by heterologous expression of Klebsiella oxytoca lysine decarboxylase," Biotechnology and Bioprocess Engineering, vol. 19, no. 6, pp. 965-972, 2014.

[24] A. Pavlova, M. Leontieva, T. Smirnova, G. Kolomeitseva, A. Netrusov, y E. Tsavkelova, "Colonization strategy of the endophytic plant growth-promoting strains of Pseudomonas fluorescens and Klebsiella oxytoca on the seeds, seedlings and roots of the epiphytic orchid, Dendrobium nobile Lindl," Journal of Applied Microbiology, vol. 123, no. 1, 217-232, 2017.

[25] C. W. Buddhi, A. Kkiu, y Y. Min-Ho, "Isolation and characterization of phosphate solubilizing bacteria (Klebsiella oxytoca) with enhanced tolerance to environmental stress," African Journal of Microbiology Research, vol. 8, no. 31, pp. 2970-2978, 2014.

[26] F. Ibañez, Z. Machado, H. Soto, E. Segal, y H. Ramos, "Endophytic and rhizospheric enterobacteria isolated from sugar cane have different potentials for producing plant growth-promoting substances," Plant Soil, vol. 353, pp. 409-417, 2012.

[27] Y. Liu, Z. Shi, L. Yao, H. Yue, H. Li, y C. Li, "Effect of IAA produced by Klebsiella oxytoca Rs-5 on cotton growth under salt stress," The Journal of General and Applied Microbiology, vol. 59, no. 1, pp. 59-65, 2013.

[28] J. D. C. Martínez-Rodríguez, M. D. L. Mora-Amutio, L. A. Plascencia-Correa, E. Audelo-Regalado, F. R. Guardado, E. Hernández-Sánchez, Y. Peña-Ramírez, A. Escalante, M. Beltrán-García, y T. Ogura, "Cultivable endophytic bacteria from leaf bases of Agave tequilana and their role as plant growth promoters," Brazilian Journal of Microbiology, vol. 45, no. 4, pp. 1333-1339, 2014.

[29] H. S. Al-Hussini, A. Y. Al-Rawahi, A. A. Al-Marhoon, S. A. Al-Abri, I. H. Al-Mahmooli, A. M. Al-Sadi, y R. Velazhahan, "Biological control of damping-off of tomato caused by Pythium aphanidermatum by using native antagonistic rhizobacteria isolated from Omani soil," Journal of Plant Pathology, vol 101, no. 2, pp. 315-322, 2018.

[30] P. Durán, J. J. Acuña, M. A. Jorquera, R. Azcón, C. Paredes, Z. Rengel, Z. y M. D. Mora, "Endophytic bacteria from selenium-supplemented wheat plants could be useful for plant-growth promotion, biofortification and Gaeumannomyces graminis biocontrol in wheat production," Biology and Fertility of Soils, vol. 50, no. 6, pp. 983-990, 2014.

[31] C. Castro, A. Hernández, L. Alvarado, y D. Flores, "DNA Barcodes in Fig Cultivars (Ficuscarica L.) Using ITS Regions of Ribosomal DNA, the psbA-trnH Spacer and the matK Coding Sequence," American Journal of Plant Science, vol. 6, pp. 95-102, 2015. 
[32] L. Alvarado-Marchena, A. Schmidt-Durán, C. Alvarado-Ulloa, R. Chacón-Cerdas, y D. Flores-Mora, "Molecular characterization of the endophytic bacteria found in the fig crops (Ficus carica var. Brown Turkey) in Costa Rica," Journal of Agricultural and Biological Science, vol. 11, no. 7, pp. 290-297, 2016.

[33] Minitab Inc, Versión 16, Estados Unidos, 2015.

[34] Tico Lab S. A., "Determinación de Glucosa: Método de Trinder," Tibás, San José, 2002.

[35] A. Zaker, M. Pazouki, y M. Vossougi, "Development of kinetic model for xanthan production in a laboratoryscale batch fermentor," Iran J Sci Technol Trans Sci, vol. 42, pp. 261-266, 2018.

[36] H. Bachmann, F. J. Bruggeman, D. Molenaar, F. B. dos Santos, y B. Teusink, "Public goods and metabolic strategies," Current Opinion in Microbiology, vol. 31, pp. 109-115, 2016.

[37] S. J. Pirt, "The maintenance energy of bacteria in growing cultures," Proc R Soc Lond Ser B Biol Sci, vol. 163, pp. 224-231, 1965.

[38] A. Quesada, "Principios de Biotecnología Microbiana," San José, Costa Rica, Editorial UCR, Pp: 60-82, 2013.

[39] S. Brisse, F. Grimont, y P. Grimont, "The Genus Klebsiella", en The Prokaryotes, Volume 6: Proteobacteria: Gamma Subclass, M. Dworkin, S. Falkow, E. Rosenberg, K. H. Schleifer, y E. Stackebrandt, eds, Springer, New York, Estados Unidos, pp. 159-196, 2006.

[40] S. Cho, K. D. Kim, J. H. Ahn, J. Lee, S. W. Kim, y Y. Um, "Selective production of 2,3-butanediol and acetoin by Newly isolated bacterium Klebsiella oxytoca M1," Appl Biochem Biotechnol, vol. 170, pp.1922-1933, 2013.

[41] J. H. Cho, C. Rathnasingh, H. Song, B. W. Chung, H. J. Lee, y D. Seung, "Fermentation and evaluation of Klebsiella pneumoniae and K. oxytoca on the production of 2,3-butanediol," Bioprocess Biosyst Eng, vol. 35, pp. 1081-1088, 2012.

[42] M. L. Alvarado-Gutiérrez, N. Ruiz-Ordaz, J. Galíndez-Mayer, E. Curiel-Quesada, y F. Santoyo-Tepole, "Degradation kinetics of carbendazim by Klebsiella oxytoca, Flavobacterium johnsoniae, and Stenotrophomonas maltophilia strains," Environmental Science and Pollution Research, vol. 27, pp. 28518-28526, 2020.

[43] P. Tang, Y. Hseu, H. Chou, K. Huang, y S. Chen, "Proteomic analysis of the effect of cyanide on Klebsiella oxytoca," Curr microbial, vol. 60, pp. 224-228, 2010.

[44] X. J. Ji, H. Huang, J. Du, J. G. Zhu, L. J. Ren, N. Hu, y S. Li, "Enhanced 2,3-butanediol production by Klebsiella oxytoca using a two-stage agitation speed control strategy," Bioresource Technology, vol. 100, pp. 3410-3414, 2009.

[45] S. Cho, T. Kim, H. M. Woo, Y. Kim, J. Lee, y Y. Um, "High production of 2,3-butanediol from biodiesel-derived crude glycerol by metabolically engineered Klebsiella oxytoca M1," Biotechnol Biofuels, vol. 8, no. 146, pp. 1-12, 2015.

[46] G. Buitrago, A. M. Otálvaro, y P. G. Duarte, "Evaluación de la transferencia de oxígeno en un biorreactor convencional con aireador externo," Rev Colomb Biotecnol, vol. 15, no. 2, pp. 106-114, 2013.

[47] N. González, J. Zamora, L. Ramos, E. Pérez, C. Pérez, y E. Salazar, "Transferencia y consumo de oxígeno en el cultivo de alta densidad del microorganismo con actividad bionematicida Tsukamurella paurometabola, C924," Revista Tecnología Química Edición Especial, pp. 162-168, 2009.

[48] L. Montoya-Pérez, y E. Durán-Herrera, "Producción de hidrógeno a partir de la fermentación de residuos agroindustriales de la piña," Tecnología en Marcha, vol. 30, no.3, pp. 106-118, 2017.

[49] J. Concha, A. Guevara, y M. Araujo, "Obtención de polvo de papaya de monte (Carica pubescens) por atomización," Revista Ingeniería UC, vol. 9, no. 1, 2002. 